\title{
Many disorders of one heart: Accidentally discovered aortic dissection, bicuspid aortic valve and hypertrophic cardiomyopathy in young patient with hypertension
}

\author{
Monika Gawor ${ }^{1}$, Mateusz Śpiewak², Łukasz Mazurkiewicz ${ }^{1,2}$, \\ Magdalena Marczak ${ }^{2}$, Ilona Michałowska ${ }^{3}$, Jacek Grzybowski ${ }^{1}$ \\ ${ }^{1}$ Department of Cardiomyopathy, Institute of Cardiology, Warsaw, Poland \\ ${ }^{2}$ Cardiac Magnetic Resonance Unit, Institute of Cardiology, Warsaw, Poland \\ ${ }^{3}$ Department of Radiology, Institute of Cardiology, Warsaw, Poland
}

A 29-year-old male with history of hypertension, hospitalized for few days in a local hospital due to pneumonia, was referred to our institution with suspicion of myocarditis and hypertrophic cardiomyopathy. On admission the patient suffered from dyspnea and cough. He denied chest pain. Physical examination revealed only bilateral rales. The baseline level of $\mathrm{C}$-reactive protein was 4.4 (range 0-0.5) $\mathrm{mg} / \mathrm{dL}$, high-sensitivity cardiac troponin T was 324 (range $0-14$ ) $\mathrm{ng} / \mathrm{L}$, and $\mathrm{N}$-terminal pro-B-type natriuretic peptide (NT-proBNP) was 3285 (range $0-125$ ) pg/mL. The standard 12-lead electrocardiogram demonstrated sinus rhythm, left atrial enlargement and left ventricular (LV) hypertrophy with non-specific ST segment and T-wave abnormalities (Fig. 1A). The chest X-ray showed bilateral lung consolidation and pulmonary congestion (Fig. 1B). Transthoracic echocardiography re- vealed significant asymmetric LV hypertrophy with preserved LV ejection fraction, bicuspid aortic valve with moderate regurgitation, and mild dilatation of the ascending aorta $(41 \mathrm{~mm})$. Due to suspicion of myocarditis, cardiac magnetic resonance imaging was performed. Hypertrophic cardiomyopathy with a maximal $\mathrm{LV}$ wall thickness of $32 \mathrm{~mm}$ and increased myocardial mass (LV mass index $149 \mathrm{~mL} / \mathrm{m}^{2}$, range $68-103 \mathrm{~mL} / \mathrm{m}^{2}$ ) was demonstrated. Moreover ascending aortic dissection was detected (Fig. 1C, D). Computed tomography confirmed aortic dissection originated in the aortic root and involving descending aorta (DeBakey Type I) (Fig. 1E, F). Patient underwent the Bentall procedure and subsequently recovered well from surgery. Aortic dissection typically presents with tearing chest pain and severe hemodynamic compromise. Painless dissection, as in this case, is relatively rare.

Conflict of interest: None declared

Address for correspondence: Monika Gawor, MD, PhD, Department of Cardiomyopathy, Institute of Cardiology, ul. Alpejska 42, 04-628 Warszawa, Poland, tel: +48 22 3434671, fax: +48 22 3434515, e-mail: mgawor@ikard.pl 


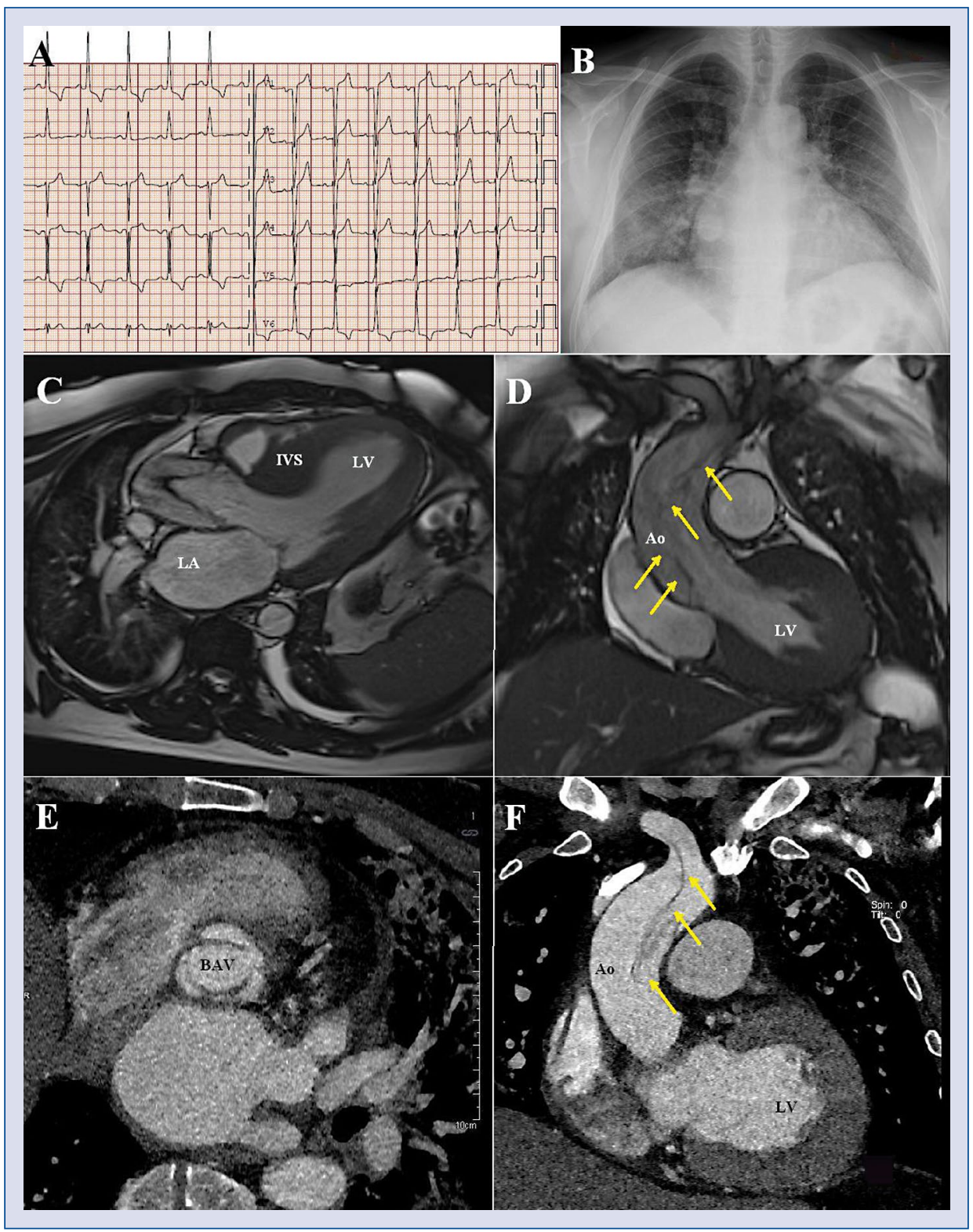

Figure 1. A. Standard 12-lead electrocardiogram; B. Chest X-ray; C. Cardiac magnetic resonance scans showing hypertrophic cardiomyopathy and D - aortic dissection; E. Computed tomography scans showing bicuspid aortic valve and F - aortic dissection. Ao - aorta; BAV - bicuspid aortic valve; LA — left atrium; LV - left ventricle; IVS - interventricular septum; arrows show dissection flap. 